\title{
New Denoising Unsharp Masking Method for Improved Intima Media Thickness Measurements with Active Contour Segmentation
}

\author{
Asraf Mohamed Moubark, David M. J Cowell, Sevan Harput and Steven Freear \\ Ultrasound Group, School of Electronic and Electrical Engineering, University of Leeds, UK. \\ E-mail: elamm@leeds.ac.uk and S.Freear@leeds.ac.uk
}

\begin{abstract}
The semi-automated balloon snake active contour (BSAC) based segmentations play a vital role in determining the intima-media thickness (IMT) for accessing the risk related to cardio vascular diseases (CVD). However, the speckle and clutter noise in the ultrasound B-mode images are known to interfere with the contour formation during segmentation. Both noise sources act as false external energy in BSAC and thus influence the resulting boundary definition. A high number of iterations are needed for the BSAC to settle on the intended boundary and in worse case totally miss the boundary when the noise presents. Thus in this work we have applied the new denoising unsharp masking (UM) method in order to reduce clutter noise in the B-mode image before the segmentation process takes place for faster and accurate measurement.
\end{abstract}

\section{INTRODUCTION}

The measurement of IMT on common carotid artery is a common clinical practice which has been used to determine the extent of plaque buildup on the walls of the arteries [1], [2]. This clinical procedure has been used by the physicians to assess risk factors or as an earlier indicator of cardiovascular diseases (CVDs) such as heart attack and stroke. This is because there is a strong link between the IMT and cardiovascular events. Thus, continuously monitoring the characteristic changes of the artery wall is crucial. According to the European Guidelines on cardiovascular disease presentation in clinical practice 2016, an IMT superior to $0.9 \mathrm{~mm}$ was considered to be abnormal and needs further medical attention immediately [2], [3]. The probability of having CVDs is more than $94 \%$ if the IMT is greater than $1.15 \mathrm{~mm}$ [1]. Ultrasound B-mode imaging has been used widely to estimate the IMT due to its simplicity and non-invasive nature [3].

The most popular techniques used for segmentation process are manual delineation of the borders or semi-automated methods such as edge detection, BSAC, level sets and Hough transform [4], [5]. The manual process of determining the borders is known to vulnerable and prone to human errors. Large variability on the IMT reading with manual techniques is subject to different experts and different equipment. The main advantage of semi-automated segmentation techniques is its accuracy in defining the seeding boundary compared to the manual and complete automated segmentation methods.

In order to measure the IMT, intima-media regions need to be segmented first. The segmentation process is based on identifying the edge or the boundary of the intima-media walls. If any noise is present between those walls, more iterations may be needed for the initial contour to reach the intended boundary in the BSAC segmentation process. At the same time, there is also a chance for false border detections. The semi-automated method relies on the quick and simple interactive initialization of BSAC aiming to increase the overall robustness to reduce errors that may be introduced by complete automatic techniques [6]. For the semi-automated BSAC, the contour is initialized by the user near to those walls. This is to reduce errors where the snake (referring to the initial contour) fails to converge or diverge to the intended borders [7].

Increasing the B-mode image contrast and spatial resolution could be beneficial for measuring the anatomical structure more accurately. By applying the proposed UM method on compound plane wave imaging (CPWI), the side lobes and clutter noise inside the anechoic regions will improve the Bmode image contrast ratio and improve the semi-automated BSAC segmentation process for better IMT measurements.

\section{Materials And Methods}

\section{A. Unsharp Masking}

The new proposed UM method taking place on the envelope of the beamformed RF signal has an iterative scheme that computes the successive new improved envelope signal as given by:

$u_{j}(x, z)=\left\{\begin{array}{l}u_{j-1}+\lambda_{\mathrm{e} 1}\left(u_{j-1}-u_{j-1}^{*}\right), u_{j-1}-u_{j-1}^{*} \geq 0 \\ u_{j-1}+\lambda_{\mathrm{e} 2}\left(u_{j-1}-u_{j-1}^{*}\right), u_{j-1}-u_{j-1}^{*}<0\end{array}\right.$

where $j$ is the number of iterations, $u_{j}(x, z)$ is the unsharp masked image, $u_{j}^{*}(x, z)$ is the original image, $\lambda_{e 1}$ and $\lambda_{e 2}$ indicate two different weightages for positive and negative errors. Two different weightage schemes have been used in the proposed UM method to selectively control the amount of intensity attenuation or amplification in region of interest (ROIs). The two main objectives for the proposed UM method are to improve the $\mathrm{B}$-mode image lateral resolution and reduce 
clutter noise mainly inside the anechoic region. This has been done by evaluating the error polarities produced between the coherent and non-coherent signals. The UM process starts with subtracting the coherent CPWI signal with noncoherent CPWI signal before log compression. The errors are then multiplied with different weightages according to their polarities. The weightages $\lambda_{1}=0.77$ and $\lambda_{2}=0.07$ were determined empirically. This arrangement of weightages is to control the attenuation level of clutter noise. Applying same weightages for both positive and negative errors will lead to a higher dynamic range and turn the grey speckle parts to black regions. This can be misidentified as anechoic regions. The value of 0.07 was assigned for the negative errors because the difference between the non-coherent CPWI and CPWI signals was already huge. This small scale was enough to attenuate the noise level. The scaled errors were added back to coherent CPWI signals and all the procedures were repeated for three times. Beyond three iterations, the quality of the Bmode image was worse with a very low level of CNR.

The received radio frequency $(\mathrm{RF})$ echoes were beamformed with delay-and-sum (DAS) before denoised with UM. The beamformed and denoised B-mode images were despeckled with the 2-D adaptive median filter with the kernel size of [19x19] to smoothen speckle and reduce clutter noise that is present in the B-mode image. A ROI with a size of $2 \mathrm{~mm}$ x $5 \mathrm{~mm}$ (width $\mathrm{x}$ length) was selected from the far wall of the common carotid artery to start segmentation before the IMT measurement. The IMT thickness is computed between the two boundaries, intima-media, at all points along the far arterial wall in the lateral direction segmented by BSAC. The difference between the upper (intima) and lower (media) pixel values was converted into values in $\mathrm{mm}$.

\section{B. Balloon Snake Active Contour}

The objective of the BSAC is to minimize the combined energy of internal (shape of the contour) and the external (the image gradient) by continuously moving itself under a certain number of iterations [8]. The overall snake energy is given as follows:

$$
E(V(s))=E_{\text {int }}(V(s))+E_{\text {ext }}(V(s))
$$

where $V(s)$ is snake contour, $E_{\text {int }}$ and $E_{\text {ext }}$ are the internal and external energy of the snake. The external energy attracts the snake to the edges and this can be represented by the following equation:

$$
E_{\text {ext }}\left(V(s)=-\| \nabla\left[G[x, z] * h[x, z] \|^{2}\right.\right.
$$

Where $G[x, z]$ is a Gaussian smoothing filter and $h[x, z]$ is the B-mode image after denoised with UM and later converted into digital formats. Note that the expression has a negative sign associated with it. The reason for this is that the internal energy of the contour needs to coincide with the external energy represented by the gradient. During each of the iterations, the overall BSAC energy is computed so that it is always the minimum.
TABLE I

EXPERIMENT PARAMETERS.

\begin{tabular}{lc}
\hline \hline Properties & Values \\
\hline Transducer & Verasonics L11-4 \\
Speed of Sound & $1540 \mathrm{~m} / \mathrm{s}$ \\
Number of Elements & 128 \\
Transducer Centre Frequency & $7.55 \mathrm{MHz}$ \\
Transducer Bandwidth $(-6 \mathrm{~dB})$ & $90.8 \%$ \\
Sampling Frequency for Tx/Rx & $160 / 80 \mathrm{MHz}$ \\
Excitation Signal & 2-Cycle Sinusoid \\
Excitation Signal Window & Tukey $(\alpha=0.5)$ \\
\hline \hline
\end{tabular}

\section{Experiments}

The In vivo data were collected from the cross section of the right CCA of a healthy volunteer. The protocol used in this study to measure the artery IMT was according to the clinical practice [9], [10]. The IMT measurements were obtained with the volunteer lying in the supine position. Three CCA samples were collected in sequence at the same posture classified as sample 1, 2 and 3. A 128-element linear array transducer (L114, Verasonics, Inc. United State of America) with a centre frequency of $7.55 \mathrm{MHz}$ and a $-6 \mathrm{~dB}$ bandwidth of $90.8 \%$ was used to collect all the data. A two-cycle sinusoidal excitation signal with a centre frequency of $7.55 \mathrm{MHz}$ was digitised with the ultrasound array research platform II (University of Leeds, UK) [11], [12]. A pulse sequence consisting of 13 angles was used with an increment of $2^{\circ}$ within a $\pm 12^{\circ}$ sector. The maximum imaging depth was set to $30 \mathrm{~mm}$. The FR is $2 \mathrm{KHz}$. The received signals were sampled at $80 \mathrm{MHz}$ before beamformed with DAS. The experimental parameters are provided in Table I. No apodization was applied to the elements along the lateral direction during transmission and reception. The thickness between the two boundaries, intimamedia is computed at all points (pixels) along the arterial far wall to obtain the IMT values.

\section{RESULTS}

The results for IMT measurements with BSAC using different iterations on DAS and UM-DAS are shown in Fig. 1. The $\mathrm{IMT}_{\text {mean }}$ value for DAS increases from $0.4002 \mathrm{~mm}$ to $0.6315 \mathrm{~mm}$ when the iterations change from 50 to 200. There is a $57.8 \%$ increment in $\mathrm{IMT}_{\text {mean }}$. This shows that the BSAC internal contour keeps deforming and has not reached the final minimum energy yet. The reason for this is clutter noise that tends to stop the internal contour from deforming by providing false edges. Denoising clutter noise with UM and applying despeckeling will make the initial contour to expand faster to the global edge. As shown in Fig. 1(d), with 50 iterations, the BSAC initial contour approaches the adventitia border much closer by combining UM and DAS. Whereas with DAS alone, as shown in Fig. 1(c), the BSAC initial contour stops at the region of clutter noise rather than on the adventitia border. Although when combining UM and DAS the contour reaches closer to adventitia border, the final border is not reached due to a low number of iterations of 50 . This can be seen in 
(a)

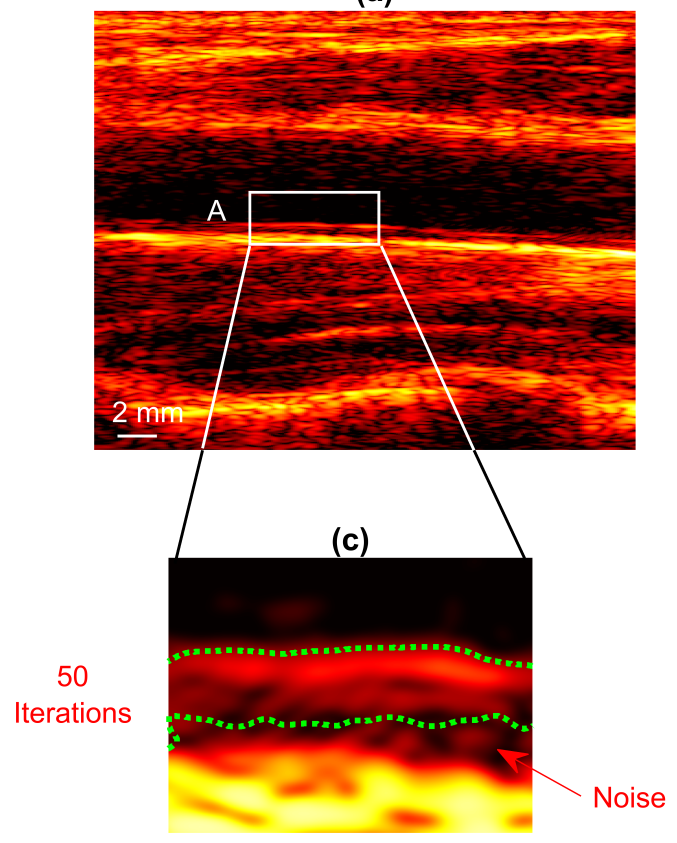

(e)

100 Iterations

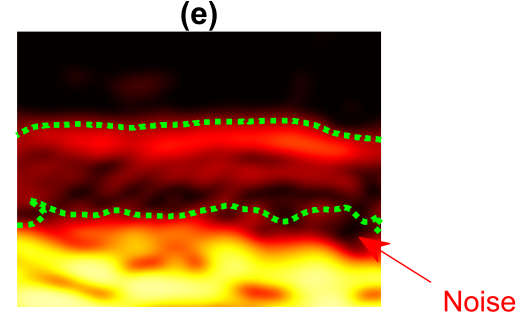

(g)

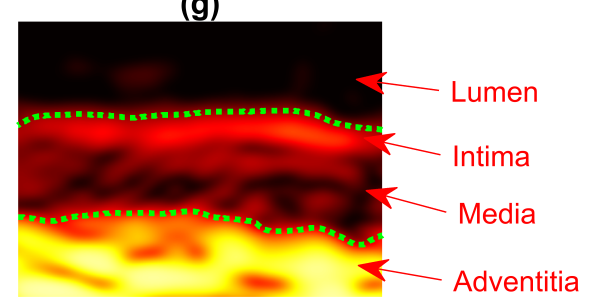

(b)

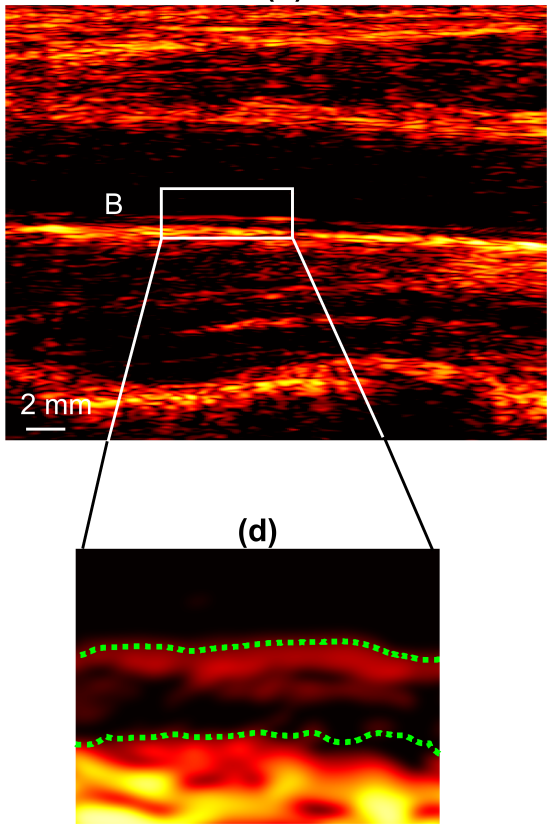

(f)

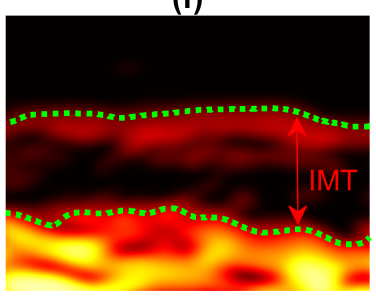

(h)

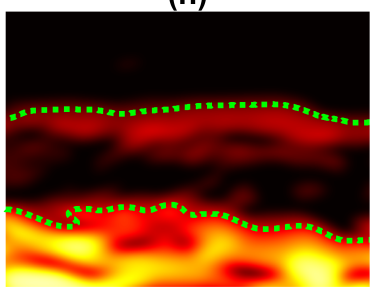

Fig. 1. The CCA beamformed with a) DAS and denoised with b) UM-DAS. The BSAC contour (green line) implemented within the ROI (white box, A (DAS) and B (UM-DAS)) after 50 iterations (c and d), 100 iterations (e and f) and 200 iterations ( $\mathrm{g}$ and $\mathrm{h}$ ). The SAC contour settles on the media border within 100 iterations for UM-DAS while it needs 200 iterations for DAS.

Fig. 1(d) as highlighted by the arrow. The difference for the $\mathrm{IMT}_{\text {mean }}$ from the 50 to 200 iterations using UM-DAS is only $18.2 \%$. This is much lower when compared to the difference of $57.8 \%$ in $\mathrm{IMT}_{\text {mean }}$ using DAS. With only 100 iterations, the segmentation process with UM techniques have reached the final border (minimum energy), and when the iteration is increased to 200, the changes of the $\mathrm{IMT}_{\text {mean }}$ between 100 and 200 iteration are small. The $\mathrm{IMT}_{\text {mean }}$ measured with UMDAS shows the difference of $0.0012 \mathrm{~mm}$ or $0.19 \%$, while DAS shows $0.1186 \mathrm{~mm}$ or $23.1 \%$ difference between 100 and 200 
TABLE II

THE IMT WITH DIFFERENT BSAC ITERATIONS

\begin{tabular}{lccc}
\hline \hline & 50 & 100 & 200 \\
\hline DAS & $0.4002 \pm 0.054$ & $0.5129 \pm 0.0466$ & $0.6315 \pm 0.046$ \\
UM-DAS & $0.5348 \pm 0.0357$ & $0.6313 \pm 0.0572$ & $0.6325 \pm 0.0612$ \\
\hline
\end{tabular}

TABLE III

THE PROCESSING TIME FOR BSAC SEGMENTATION WIHT DIFFERENT ITERATIONS.

\begin{tabular}{lccc}
\hline \hline & 50 & 100 & 200 \\
\hline DAS & 2.4 & 3.04 & 4.36 \\
UM-DAS & 2.39 & 3.28 & 4.39 \\
\hline
\end{tabular}

iterations.

The processing time for BSAC segmentation is not affected by the techniques investigated but dependent on the total number of iterations. The processing time for 50, 100 and 200 iterations for both DAS and UM-DAS are almost the same and given in Table III. The number of iterations is set according to the initial contour size [13]. The definition of the initial contour is dependent on the object shape and in general drawn as near as possible to the intended border to reduce the number of iterations.

\section{CONCLusion}

The proposed UM as a denoising tool for DAS is able to reduce clutter noise in between the intima-media layers and improve the IMT measurement. The number of iterations needed for BSAC to settle on the final border is less with UM (100 iterations) compared to that without the denoising technique. The UM techniques is able to provide better results with less time in measuring the IMT compared to that using DAS. One of the challenges during the IMT measurement on the B-mode image with BSAC is the placement of the initial contours. The manual placement of the initial contour should be as near as possible to the intima and adventitia borders in the ROI.

\section{REFERENCES}

[1] R. T. Hurst, D. W. Ng, C. Kendall, and B. Khandheria, "Clinical use of carotid intima-media thickness: review of the literature," Journal of the American Society of Echocardiography, vol. 20, no. 7, pp. 907-914, 2007.

[2] F. Hobbs, M. Piepoli, A. Hoes, S. Agewall, C. Albus, C. Brotons, A. Catapano, M. Cooney, U. Corra, B. Cosyns et al., "2016 european guidelines on cardiovascular disease prevention in clinical practice." European heart journal, vol. 37, no. 29, pp. 2315-2381, 2016.

[3] J. Xu and Y. Cao, "Radiation-induced carotid artery stenosis: a comprehensive review of the literature," Interventional Neurology, vol. 2, no. 4, pp. 183-192, 2013.

[4] C. P. Loizou, C. S. Pattichis, C. I. Christodoulou, R. S. Istepanian, M. Pantziaris, and A. Nicolaides, "Comparative evaluation of despeckle filtering in ultrasound imaging of the carotid artery," IEEE transactions on ultrasonics, ferroelectrics, and frequency control, vol. 52, no. 10, pp. 1653-1669, 2005.

[5] C. P. Loizou, "A review of ultrasound common carotid artery image and video segmentation techniques," Medical \& Biological Engineering \& Computing, vol. 52, no. 12, pp. 1073-1093, Dec 2014. [Online]. Available: https://doi.org/10.1007/s11517-014-1203-5
[6] G. Zahnd, M. Orkisz, A. Sérusclat, P. Moulin, and D. Vray, "Simultaneous extraction of carotid artery intima-media interfaces in ultrasound images: assessment of wall thickness temporal variation during the cardiac cycle," International Journal of Computer Assisted Radiology and Surgery, vol. 9, no. 4, pp. 645-658, 2014.

[7] C. P. Loizou, A. Nicolaides, E. Kyriacou, N. Georghiou, M. Griffin, and C. S. Pattichis, "A comparison of ultrasound intima-media thickness measurements of the left and right common carotid artery," IEEE Journal of Translational Engineering in Health and Medicine, vol. 3, pp. 1-10, 2015.

[8] F. Shariat, "Object segmentation using active contours: A level set approach," 2009.

[9] I. B. Casella, C. Presti, R. M. P. Porta, C. R. D. Sabbag, M. A. Bosch, and Y. Yamazaki, "A practical protocol to measure common carotid artery intima-media thickness," Clinics, vol. 63, no. 4, pp. 515-520, 2008.

[10] V. D. Garovic, N. M. Milic, T. L. Weissgerber, M. M. Mielke, K. R. Bailey, B. Lahr, M. Jayachandran, W. M. White, H. N. Hodis, and V. M. Miller, "Carotid artery intima-media thickness and subclinical atherosclerosis in women with remote histories of preeclampsia: Results from a rochester epidemiology project-based study and meta-analysis," Mayo Clinic Proceedings, vol. 92, no. 9, pp. 1328 - 1340, 2017. [Online]. Available: http://www.sciencedirect.com/science/article/pii/S0025619617304378

[11] D. Cowell and S. Freear, "Quinary excitation method for pulse compression ultrasound measurements," Ultrasonics, vol. 48, no. 2, pp. 98-108, 2008.

[12] P. R. Smith, D. M. J. Cowell, B. Raiton, C. V. Ky, and S. Freear, "Ultrasound array transmitter architecture with high timing resolution using embedded phase-locked loops," IEEE Transactions on Ultrasonics, Ferroelectrics, and Frequency Control, vol. 59, no. 1, pp. 40-49, January 2012.

[13] C.-y. Yu, W.-s. Zhang, Y.-y. Yu, and Y. Li, "A novel active contour model for image segmentation using distance regularization term," Computers \& Mathematics with Applications, vol. 65, no. 11, pp. 1746-1759, 2013. 